\title{
Mathematical Modelling for Keyhole Surgery Simulations: Spleen Capsule as an Elastic Membrane
}

\author{
PENNY J. DAVIES ${ }^{\mathrm{a}, *}$, FIONA J. CARTER $^{\mathrm{b},}{ }^{\dagger}$, DAVID G. ROXBURGH ${ }^{\mathrm{c},} \neq$ and ALFRED CUSCHIERI ${ }^{\mathrm{d}}, \mathbb{1}$

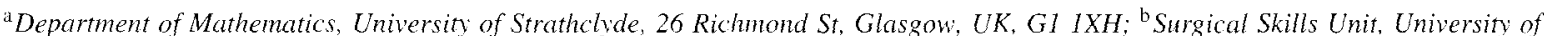 \\ Dundee, Level 5, Ninewells Hospital, Dundee, UK, DD1 9SY; 'Department of Mathematics, Heriot-Wat University, Riccarton, \\ Edinburgh, UK, EHI4 4AS; 'Department of Surgery, University of Dundee, Level 6, Ninewells Hospital, Dundee, UK, DDI 9SY
}

(Received 22 October 1997; Revised 31 March 1998; In final form 26 June 1998)

\begin{abstract}
We show that the mechanical properties of the outer covering (or capsule) of pig spleen can be modelled as a nonlinear elastic membrane with an exponential stress-strain law. Knowledge of the capsule's elastic properties is important for the development of a virtual reality training package for keyhole surgery. Because capsule tissue is very fragile and tears when clamped and pulled it is not possible to obtain experimental data for which the usual uniform stretch data-fitting approach can be adopted. Instead we perform experiments in which the capsule deforms non-uniformly and describe how numerical solutions of the corresponding boundary value problem can be fitted to the experimental data in order to validate the biomechanical model.
\end{abstract}

Keywords: Nonlinear elasticity, biomechanics, membrane, modelling

Classification Categories: AMS(MOS) subject classification: 73C50, 73K10, 73P05, 92C10

\section{INTRODUCTION}

'Keyhole' (or laparoscopic) surgery is becoming increasingly popular because it is both beneficial to patients and cost-effective. A typical laparoscopic operation involves puncturing the abdominal wall in four or five places, and inserting ports through which are put a viewing telescope and various $5 \mathrm{~mm}$ surgical instruments. The surgeon uses the instruments to perform the operation whilst viewing the telescopic image via an attached camera and light source connected to a high quality video monitor (the camera is usually controlled by an assistant). The main benefits to the patient compared to conventional (or 'open') surgery include quicker recovery, early discharge from hospital, reduced

\footnotetext{
${ }^{*}$ Corresponding Author: E-mail: penny.davies@strathac.uk

${ }^{\dagger}$ E-mail: fjcarter@ninewells.dundee.ac.uk

E-mail: davidr@ma.hw.ac.uk

IE-mail: a.cuschieri@dundee.ac.uk
} 
period of short-term disability and minimal scarring because of the small wounds.

The surgical techniques needed to perform this type of operation are very different from those needed for conventional surgery. The main additional difficulties are that

- there is a limited field of view, and the surgeon operates from a two dimensional image; and

- the surgical instruments are slender and long $(30 \mathrm{~cm})$ so there is much less tactile feedback (Brown, Satava and Rosen 1994).

Thus successful endoscopic surgery requires the surgeon to be skilled in the manipulation of small instruments under guidance of the endoscopic image on a video monitor, and these skills require formal, specialised training. Centres exist to run training courses in these techniques. The courses typically consist of a range of activities including watching videos of (both successful and unsuccessful) operations, 'live' video links to operations in progress, and gaining practical experience on restructured animal tissue or synthetic models (Carter, Russell, Dunkley and Cuschieri 1994). Whilst excellent basic training can be achieved by these means, neither the restructured nor synthetic tissues are completely realistic since they do not bleed or have the same handling characteristics as living tissue. In some countries (although not the UK) training also takes place on live animals, which has the advantage that the trainee surgeon is able to practice control of bleeding. The disadvantages are that the anatomy is different, and that it is only possible to repeat the procedure a limited number of times. There are also ethical considerations involved in using live animals for training.

It is envisaged that computer simulation of laparoscopic operations (i.e. virtual reality (VR) keyhole surgery) could provide a more realistic and flexible training environment. In order to do this the simulation package will have to fulfil the following criteria (Satava 1993):

1. the VR surgical instruments must interact with the VR organs and tissue in a physically realistic way. In particular, organs must deform appropriately when grasped, lifted or cut (this includes bleeding or leaking fluids);

2. what the trainee surgeon sees and feels when using the simulator must be realistic. The image must have high enough resolution to appear real, and the forces required to lift or cut the VR tissue should match those felt in reality; and

3. the properties (size, shape and condition) of the VR organs must be able to be changed in order to reflect the changes caused by disease, as well as the variability of different "normal' tissues.

If a computer simulation is to be effective then it must be based on clinical measurements. It is obviously not possible to construct a database that contains the 'result' of all possible interactions with the tissues of live human subjects. Hence probably the best solution is to use mathematical models to predict the response of tissues to a range of deformations. Many authors (for example Fung (1967, 1993), Demiray $(1972,1981,1983)$, Wang, Wang, Yan and Liu (1992), Humphrey and Yin (1989)) have examined mechanical models for various types of soft animal tissue, noting that the response of such materials is extremely nonlinear. There has been particular interest in modelling bovine pericardium tissue, which is used in the manufacture of replacement heart valves e.g. (Trowbridge, Black and Daniel 1985, Trowbridge and Crofts 1987, Zioupos, Barbenel, Fisher and Wheatley 1993, Zioupos and Barbenel 1994). A comprehensive history of biomechanical modelling in this area can be found in (Trowbridge et al. 1985).

Tissue from human spleens is similar to that from pigs (Carter et al. 1994), and Carter and Davies (1996) contains a description of how data from compression experiments for ex-vivo pig spleen is modelled extremely well by a two-parameter exponential stress-strain law developed by Fung (1967) and Demiray (1972). The spleen is completely surrounded by a tough fibrous outer membrane or capsule. The capsule mainly consists of randomly oriented elastin and collagen fibres, and plays an important role in maintaining the structure of the organ. Knowledge of its elastic properties is 
therefore essential in predicting the response of a spleen to interactions with surgical probes and other instruments. In this paper we focus on modelling the spleen capsule as an elastic membrane, and we show that the membrane version of the 3D Fung-Demiray model (Fung 1967, Demiray 1972) used for the interior tissue in (Carter and Davies 1996) predicts its elastic behaviour well.

The capsule is too fragile to be clamped and pulled as described in the classical experiments on rubber reported in (Rivlin and Saunders 1951) (it tears very easily), and so we have had to devise another way to measure its resistance to deformation (see Section 2.1). The resulting deformation is not homogeneous, and so fitting the model to the experimental data is non-trivial (it involves solving the appropriate membrane equilibrium equations numerically), but possible. The deformation is a simple type of the class considered by Roxburgh, Steigmann and Tait (1995) for a (known) MooneyRivlin material (see also Fulton and Simmonds (1986)).

The paper is organised as follows. We begin with a report of the experimental procedure and observations. Section 3 is devoted to a brief description of nonlinear elasticity and membrane theory, and the boundary value problem satisfied by the membrane corresponding to the experimental situation is derived at the start of Section 4 . The equations are similar to those studied in (Fulton and Simmonds 1986, Roxburgh et al. 1995), and in Sections 4.2-4.3 we show how the shooting method approach of Roxburgh et al. (1995) can be modified to solve them, and then present some numerical results. We conclude with a short discussion of our results.

\section{EXPERIMENTS}

We begin the section by describing how the capsule tissue is prepared and then loaded in order to obtain force-displacement data. The second subsection describes the results of varying the rate of loading the tissue, the tissue's behaviour on unloading (there is a small amount of hysteresis), and how it behaves when it is subjected to repeated loading cycles.

\subsection{Experimental Procedure}

Fresh pig spleen was obtained from a local abattoir and frozen until use. The outer capsule is strongly attached to the underlying tissue and it is difficult to remove a sizeable piece by conventional dissection methods. For these experiments the capsule was removed using an ultrasonic dissection device (Selector, Surgical Technology Group, Andover, UK) and then stored in isotonic saline solution (to prevent it drying out) until tested. It should be noted that there is not a clear distinction between capsule and underlying tissue, rather that the tissue's mechanical properties change rapidly in a thin boundary layer just below the surface. Hence the capsule samples tested were of slightly different thickness and there was some variation in the physical properties of their lower surfaces.

Membrane models for thin materials are usually obtained from data in which the deformation is a uniform stretch (Rivlin and Saunders 1951), since in this case the membrane equilibrium equations are identically satisfied and the model can be investigated by a simple best fit of parameter values to the data. When the capsule is removed from the underlying material it is very fragile, and tears if it is clamped and then stretched. This means that it is not possible to use the usual uniform data fitting approach in order to find a suitable membrane model for the capsule. However it is possible to obtain force-displacement data by clamping it securely between two square steel plates that have had a circular hole cut in them and then lowering a small circular probe into the centre of the membrane. This causes the capsule to deform into a shape that looks roughly like a truncated cone, as shown schematically in Figure 2.1. Small dots of methylene blue dye are placed around the circular edge of the exposed membrane, so that it is possible to 

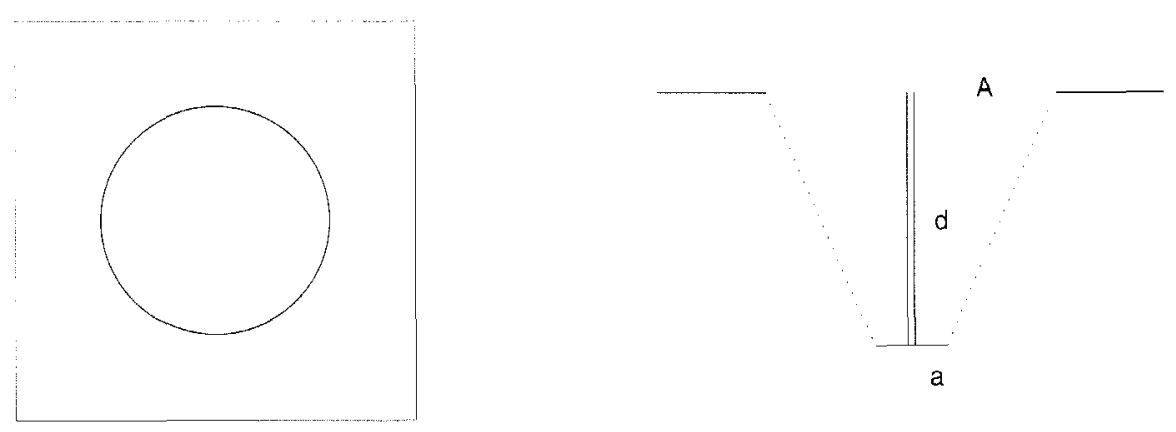

FIGURE 2.1 Schematic illustration of the force-displacement experiments performed on the outer capsule membrane. The capsule originally occupies a circle of radius $A$ in its unstressed state, clamped between two steel plates (the left hand figure is a plan view). It is deformed into a shape that looks roughly like a truncated cone (right hand figure) by displacing at circular probe of radius $a$ vertically through a distance $d$ as shown.

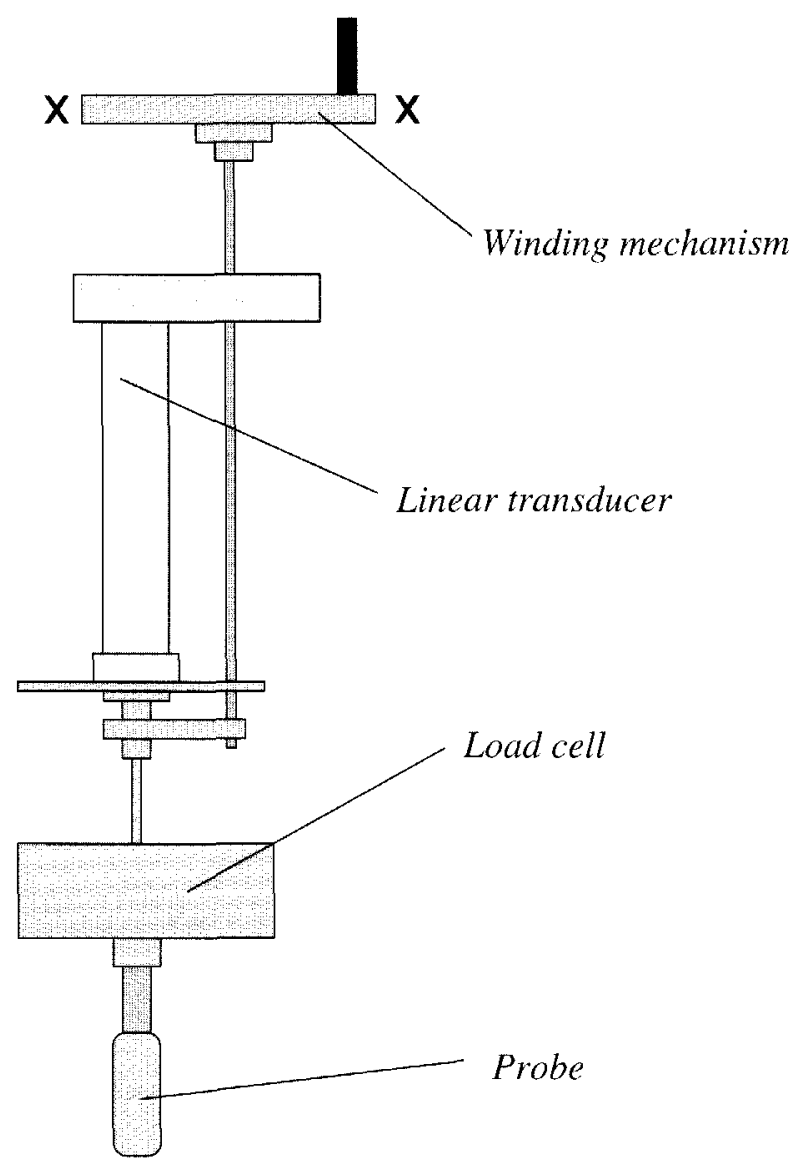

FIGURE 2.2 Schematic illustration of the testing rig used for the force-displacement capsule experiments. See text for details.

tell that the capsule is clamped securely between the plates. For all our experiments the radius of the circular hole in the plates is $A=22.35 \mathrm{~mm}$, and that of the probe is $a=2.25 \mathrm{~mm}$.
The probe is connected to a $5 \mathrm{Kg}$ load cell and linear transducer connected in series, and to a winding mechanism (Figure 2.2 shows the experimental testing rig). The position marked ' $X$ ' is clamped in 
place, and turning the winding mechanism lowers the load cell and probe into the tissue (one full rotation advances the probe $1 \mathrm{~mm}$ ). The output from the sensors is amplified and passed through an analogue to digital conversion card fitted in a computer. The data is then scaled, to give a series of force $\left(f_{k}\right)$ and displacement $\left(d_{k}\right)$ readings. The force is the total force exerted by the probe on the material, and the displacement is the vertical displacement of the probe into the tissue.

\subsection{Experimental Observations}

We have observed that the spleen capsule samples respond to loading in qualitatively the same way as reported in the literature for other animal membrane tissues, e.g. (Fung 1967, Trowbridge et al. 1985, Trowbridge and Crofts 1987, Zioupos et al. 1993) and (Fung 1993, Chapter 7). The capsule exhibits 'tissue relaxation' (Fung 1993, Trowbridge et al. 1985), that is, if the probe is depressed into the tissue and then held fixed the amount of force exerted on the probe by the tissue gradually decreases.

We are primarily interested in modelling and predicting the initial response of the capsule to a surgical probe or scalpel, and so we need to move the probe fast enough so that the tissue does not have time to relax significantly between loading increments. We found probe depression rates of 60 and $120 \mathrm{rpm}$ to be suitable for this. The mechanical response of the capsule does not seem to depend on the speed at which the probe is lowered, provided it is fast enough to prevent the tissue relaxing, as illustrated in Figure 2.3 (see also Trowbridge and Crofts (1987)). This shows force-displacement curves for 33 different capsule samples (17 at $60 \mathrm{rpm}$ and 16 at $120 \mathrm{rpm}$ ), and there is clearly more variation within the data at each rate than between data obtained at the two different rates.

The capsule exhibits a small amount of hysteresis - the loading and unloading forcedisplacement curves are different, as shown in

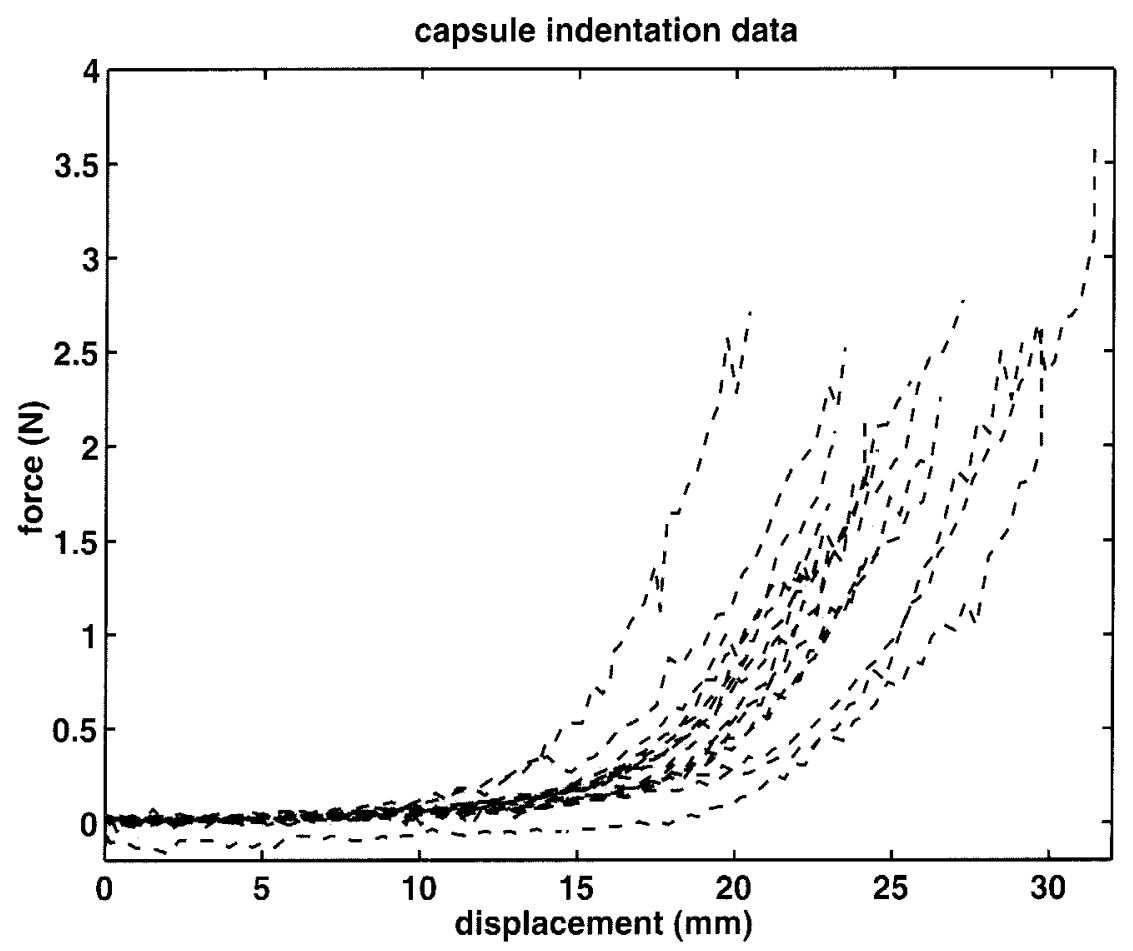

FIGURE 2.3 Force-displacement plots obtained for various capsule samples. The probe is advanced at a rate of $60 \mathrm{rpm}$ (solid grey) and $120 \mathrm{rpm}$ (dashed black). 

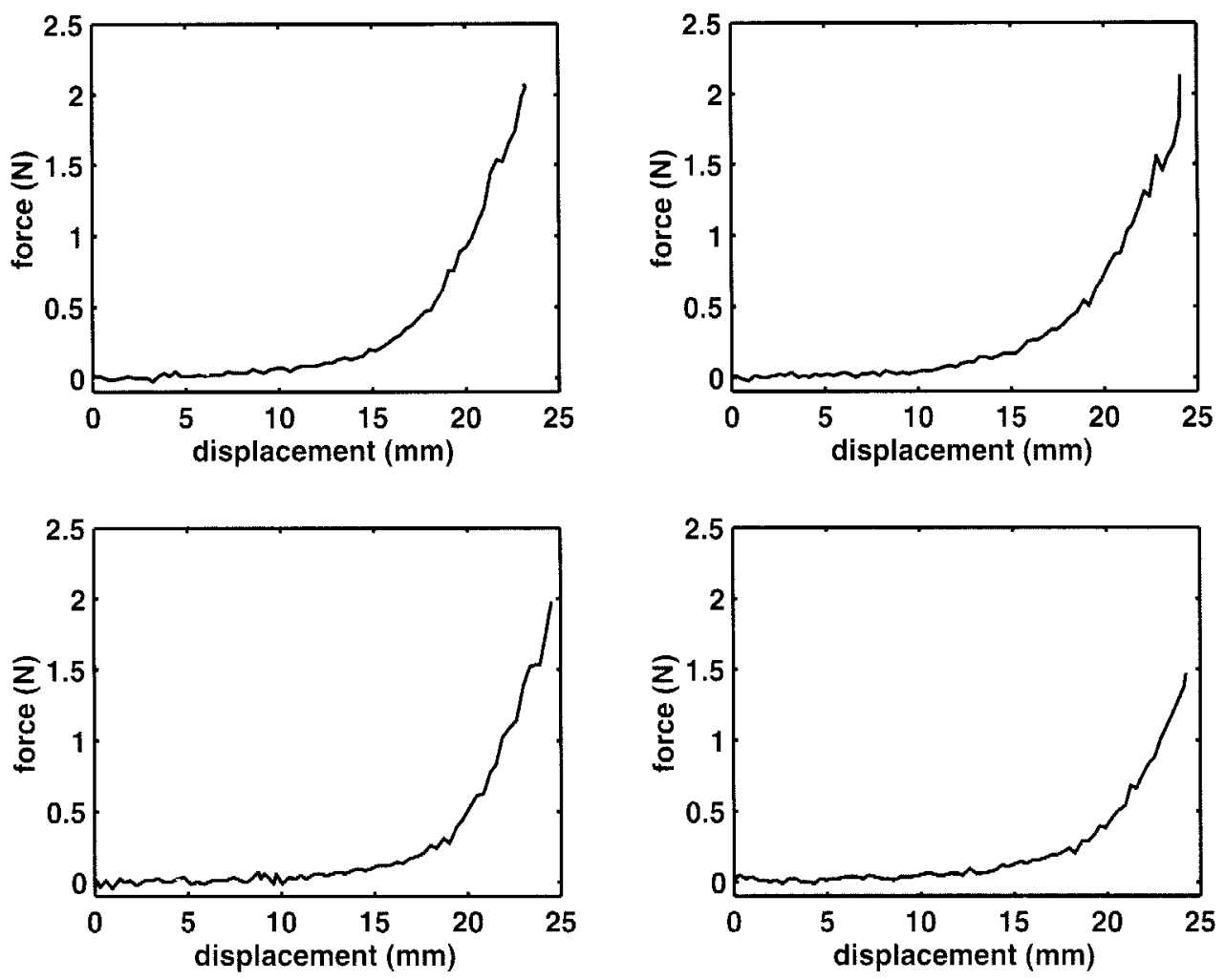

FIGURE 2.4 Force-displacement graphs showing hysteresis obtained for four typical capsule samples. In each plot the black line is the loading curve and the grey line is the unloading curve.

Figure 2.4. These graphs are very similar to those obtained for uniaxial stretches of rabbit mesentery in (Fung 1967), and to the many loading/unloading curves shown in (Fung 1993, Chapter 7) and (Trowbridge et al. 1985, Trowbridge and Crofts 1987). The fact that the loading/unloading curves contain a hysteresis loop means that capsule tissue is not a perfectly elastic material, and so there is no stored energy function (see Section 3) that (approximately) characterises its behaviour in all stress regimes. However, since our aim is to predict the capsule's response to initial loading, we shall seek a stored energy function that models the behaviour of the tissue shown in the black curves of Figure 2.4. If the response to primary unloading were required, then a model for this could be obtained from the unloading data (the grey curves) by using a different stored energy function. Fung (1993) terms this approach 'pseudo-elasticity'.
We have also investigated the effects of repeated loading on the capsule tissue, again with similar results to those reported elsewhere (Fung 1993, Trowbridge et al. 1985, Zioupos et al. 1993). Figure 2.5 shows the outcome of repeated loading experiments performed on four capsule samples. In each of these graphs there is a marked difference between the first loading curve (solid grey line) and the second and third curves (black dashed and dotted lines respectively). The fourth plot is somewhat anomalous in the sense that its fourth and fifth loading curves occur to the left of the first one, but this could perhaps be due to experimental error.

The reason for the difference in loading curves is discussed by Fung (1993, Chapter 7). He says that in general the loading curves shift to the right with each cycle and that if the sample is loaded and unloaded indefinitely then the difference between two successive loading curves becomes vanishingly 

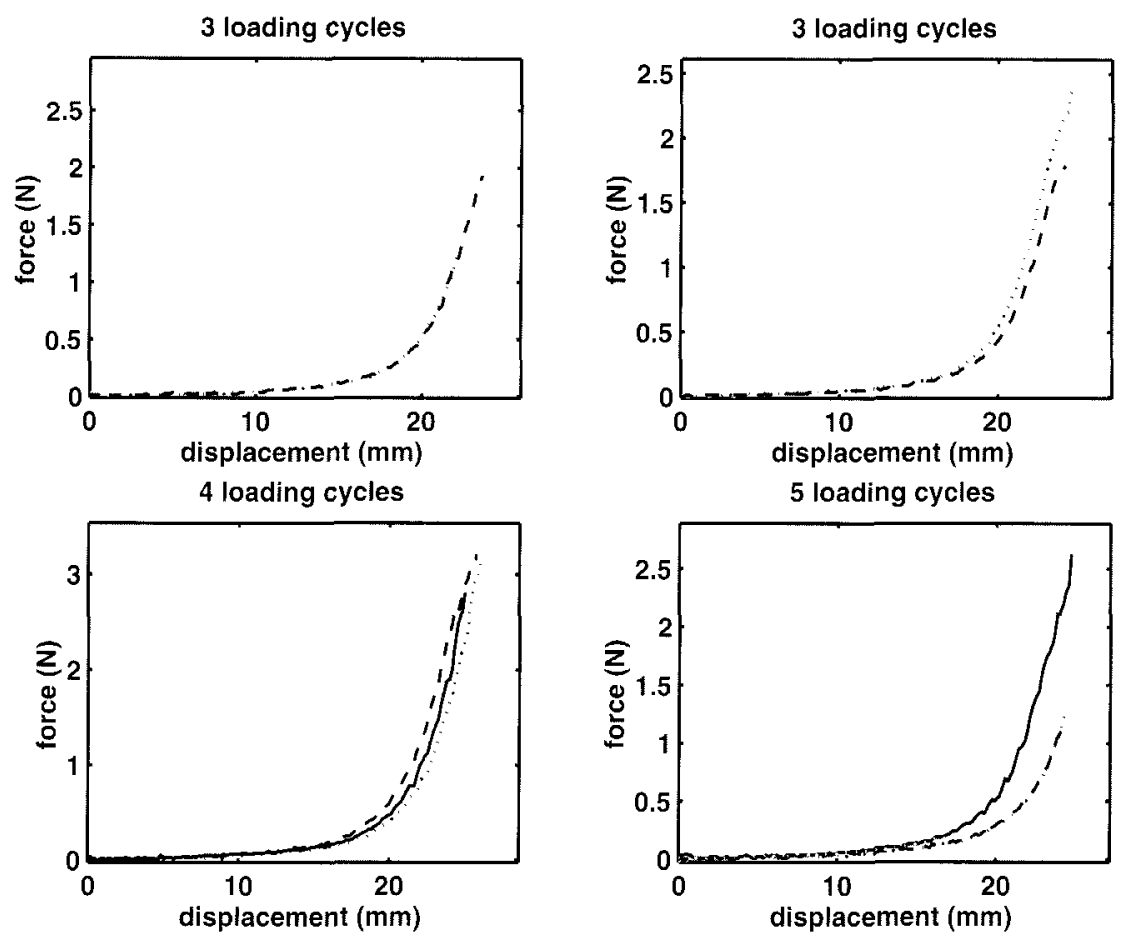

FIGURE 2.5 Force-displacement graphs obtained for four typical capsule samples showing the effects of repeated loading. Each plot contains between three and five loading curves (the number of loading cycles is given at the top of the plot) obtained in the order: (1) grey solid; (2) black dashed; (3) black dotted; (4) black solid; (5) grey dotted.

small. He calls this phenomenon 'preconditioning' and states that "The reason that preconditioning occurs in a specimen is that the internal structure of the tissue changes with the cycling. By repeated cycling, eventually a steady state is reached at which no further change will occur unless the cycling routine is changed".

Again we emphasise that it is the capsule's initial response that we are interested in and so our aim is to produce a biomechanical model for the primary loading curve and not for the loading behaviour of the preconditioned tissue. It is conceivable that there are situations in which it would be advantageous to be able to predict the behaviour of a piece of capsule tissue that had been subjected to repeated loading, and if this is the case then it would be appropriate to instead use a model for preconditioned tissue.

In summary, we have seen that

- spleen capsule exhibits tissue relaxation;

- as long as the capsule is loaded moderately quickly (so there are no noticeable relaxation effects) then the force-displacement curves seem to be independent of the loading rate;

- there is a small hysteresis loop in the loading/unloading curves; and

- repeated loading changes the tissue's mechanical properties.

These observations indicate that spleen tissue is not perfectly elastic. However, since our objective is to obtain a mathematical model that describes only the initial loading response of the capsule, we shall regard it as being a (nonlinear) elastic material and determine a mathematical model that fits the primary loading data.

\section{NONLINEAR ELASTICITY AND MEMBRANE THEORY}

Recall that our aim is to model the capsule tissue in its primary loading regime as an elastic material. The figures in the previous section show that the 
capsule stretches a relatively large amount during the experiments and that the loading curves are far from being linear. Indeed, the mathematical theory of elastic materials capable of undergoing large deformations is inherently nonlinear - that is, the stress (force per unit area) depends nonlinearly on the deformation. Comprehensive treatments of the theory are given for example in (Atkin and Fox 1980, Gurtin 1983, Ogden 1984, Beatty 1987). We begin by briefly giving some notation relating to the (static) theory of nonlinear elasticity, and then describe the basic parts of membrane theory necessary for modelling the capsule (see for example Green, Naghdi and Wainwright (1965), Naghdi (1972) for a fuller description of this). We pay particular attention to the membrane version of the three dimensional biomechanical model considered by Demiray in (1972) (this is a generalisation of the one dimensional model of Fung (1967); see also (Beatty 1987, Fung 1993. Humphrey and Yin 1989, Trowbridge et al. 1985)).

Suppose that an elastic material occupies the region $\Omega \in \mathbb{R}^{3}$ in its natural unstressed state. Points $\boldsymbol{x}=\left(x_{1}, x_{2}, x_{3}\right)=\left(x_{\alpha}\right) \in \Omega$ are called the material or reference coordinates. A deformation of $\Omega$ is a smooth one-to-one map

$$
y: \Omega \rightarrow \mathbb{R}^{3},
$$

where $\boldsymbol{y}=\left(y_{1}, y_{2}, y_{3}\right)=\left(y_{i}\right) \in \mathbb{R}^{3}$. The $3 \times 3$ matrix $F$ with components given by $F_{i, \alpha}=\partial y_{i} / \partial x_{\alpha}$ is called the deformation gradient. The principal values of $F$ (i.e. the eigenvalues of $\sqrt{F^{T} F}$ ) are called the principal stretches, and denoted by $v_{1}, v_{2}$ and $v_{3}$.

We suppose that $\Omega$ is deformed very slowly (quasistatically) so that the deformation $y$ and all forces can be assumed to be independent of time, and denote the force per unit area acting at a point $y$ on the surface of the deformed body with outward unit normal $\boldsymbol{n}$ by $\boldsymbol{s}(\boldsymbol{y}, \boldsymbol{n})-$ the Cauchy stress vector. It can be shown that $\boldsymbol{s}(\boldsymbol{y}, \boldsymbol{n})=\mathbb{T} \boldsymbol{n}$ for a symmetric $3 \times 3$ matrix $\mathbb{T}$ called the Cauchy stress tensor. The constitutive assumption of nonlinear elasticity is that the stress tensor at a point $y$ in the deformed body depends only on the material and on the deformation gradient at $y$. Although the capsule is not elastic over a complete loading/unloading cycle (Figure 2.4), we shall assume that it behaves elastically whilst being loaded and that it can be regarded as a hyperelastic material during its first loading cycle.

Spleen capsule tissue is relatively uniform, does not have a noticeable preferred direction, and it is likely that its volume changes little when it is deformed (interior spleen tissue is certainly virtually incompressible (Carter and Davies 1996)). It is therefore physically reasonable to make some further simplifying assumptions and we take it to be homogeneous, isotropic and incompressible. In this case the stress-strain relationship takes the form (see e.g. Atkin and Fox (1980))

$$
\mathbb{T}=-p \mathbf{1}+\frac{\partial W(F)}{\partial F} F^{T},
$$

where $p$ is the hydrostatic pressure (it is a Lagrange multiplier corresponding to the incompressibility constraint $\operatorname{det} F \equiv 1$ in $\Omega$ ), and $\mathbb{W}(F)$ is a scalar function called the stored energy function. Our aim is to show that the membrane version of the three dimensional model considered by Demiray in (1972) matches the biomechanical properties of the capsule well. Demiray's stored energy function is

$$
W(F)=\frac{\mu}{2 \gamma}\left\{\exp \left(\gamma\left(v_{1}^{2}+v_{2}^{2}+v_{3}^{2}-3\right)\right)-1\right\}
$$

where $\mu$ and $\gamma$ are positive constants.

We now derive a membrane model for spleen capsule based on (3.1). We assume that $\Omega$ is very thin in the $x_{3}$-direction and that its centre-plane $S$ lies flat along $x_{3}=0$ in its natural unstressed state. We shall approximate deformations of $\Omega$ by regarding $S$ as a homogeneous elastic surface (an elastic membrane) that is endowed with a function that specifies the stored energy per unit referential area. Points on the surface are represented by the reference coordinates $\boldsymbol{x}=\left(x_{1}, x_{2}\right) \in S$. In a deformation of the membrane the point originally at $x$ is displaced to the point with position $\boldsymbol{y}(\boldsymbol{x}) \in \mathbb{R}^{3}$. This deformation 
induces a natural basis $\left\{\phi_{1}, \phi_{2}\right\}$ defined by

$$
\phi_{\alpha}=\frac{\partial y}{\partial x_{\alpha}} \quad \text { for } \alpha=1,2 .
$$

The strain tensor is

$$
C=\left(C_{\alpha \beta}\right)=\left(\begin{array}{ll}
\phi_{11} & \phi_{12} \\
\phi_{21} & \phi_{22}
\end{array}\right),
$$

where $\phi_{\alpha \beta}=\phi_{\alpha} \cdot \phi_{\beta}$ for $\alpha, \beta \in\{1,2\}$. We adopt the usual constitutive assumption of homogeneous isotropic membrane theory (Naghdi 1972), namely that $W$, the stored energy per unit area of $S$, depends only on the principal invariants of $C$. The principal invariants of the strain are its trace $I_{1}=C_{11}+C_{22}$ and determinant $I_{2}=C_{11} C_{22}-C_{12} C_{21}$. Because $C$ is positive definite and symmetric it has two real positive principal values $\lambda_{1}^{2}$ and $\lambda_{2}^{2}$, where $\lambda_{1}$ and $\lambda_{2}$ are called the (membrane) principal stretches. The stored energy can also be expressed as a symmetric function of these stretches, i.e.

$$
W(C)=w\left(\lambda_{1}, \lambda_{2}\right)
$$

for some function $w$ satisfying $w\left(\lambda_{1}, \lambda_{2}\right)=w\left(\lambda_{2}, \lambda_{1}\right)$.

Following Roxburgh et al. (1995) we suppose that $\tau$ is the contact force per unit length across a material curve on $S$, and let $\nu$ be the unit normal to the curve lying in the tangent plane of $S$. Then

$$
\tau=T \nu
$$

where $T=\left[\boldsymbol{T}^{1} ; \boldsymbol{T}^{2}\right]$ and the $\alpha$ th column $\boldsymbol{T}^{\alpha}$ of $T$ is given by

$$
\boldsymbol{T}^{\alpha}=\Phi_{\alpha \beta} \phi_{\beta},
$$

for $\alpha=1,2$, where we use the summation convention that repeated indices (here $\beta$ ) are summed, and where

$$
\Phi_{\alpha \beta} \equiv \frac{\partial W}{\partial C_{\alpha \beta}}+\frac{\partial W}{\partial C_{\beta \alpha}} .
$$

(The tensor $T$ is analogous to the Piola-Kirchhoff stress of 3D elasticity, although here it gives a measure of the force per unit length rather than per unit area.)

The elastostatic membrane equations in the absence of internal forces are

$$
\boldsymbol{T}_{, \alpha}^{\alpha}=\mathbf{0} .
$$

These can be written in terms of $w$ by using (3.3) and the identities

$2 \lambda_{1}^{2}=I_{1}+\left(I_{1}^{2}-4 I_{2}\right)^{1 / 2}, 2 \lambda_{2}^{2}=I_{1}-\left(I_{1}^{2}-4 I_{2}\right)^{1 / 2}$,

and

$$
\frac{\partial W}{\partial C_{\alpha \beta}}=\frac{\partial w}{\partial \lambda_{1}} \frac{\partial \lambda_{1}}{\partial C_{\alpha \beta}}+\frac{\partial w}{\partial \lambda_{2}} \frac{\partial \lambda_{2}}{\partial C_{\alpha \beta}} .
$$

Here we investigate the membrane model that is analogous to the 3D model (3.1). Incompressibility implies that the principal stretches of three dimensional deformations satisfy $v_{1} v_{2} v_{3}=1$. The stretches $v_{1}$ and $v_{2}$ are approximated by the membrane stretches $\lambda_{1}$ and $\lambda_{2}$ respectively, and so $v_{3}$ is approximately equal to $\left(\lambda_{1} \lambda_{2}\right)^{-1}$. Substituting for the $v_{i}$ 's in the $3 \mathrm{D}$ stored energy function (3.1) yields the membrane model

$$
w\left(\lambda_{1}, \lambda_{2}\right)=\frac{\mu}{2 \gamma}\left\{\exp \left(\gamma\left(I_{1}+1 / I_{2}-3\right)\right)-1\right\},
$$

where $I_{1}=\lambda_{1}^{2}+\lambda_{2}^{2}$ and $I_{2}=\lambda_{1}^{2} \lambda_{2}^{2}$.

This is the membrane stored energy that we use for the capsule during its first loading cycle. In the rest of the paper we show how to calculate the values of the parameters $\gamma$ and $\mu$ that best match our experimental data (in a least squares sense), and demonstrate that this 'best fit' model gives a good representation of the initial mechanical behaviour of capsule tissue.

\section{THE BOUNDARY VALUE PROBLEM}

In this section we describe how experimental data can be used to investigate the validity of the membrane stored energy function (3.5). We begin by detailing an appropriate boundary value problem that corresponds to the deformation of the capsule during the experiments described in Section 2. Then in Section 4.2 we show how the shooting method approach used by Roxburgh et al. (1995) can be modified to give a numerical algorithm that computes the values of the material parameters $\gamma$ and $\mu$ for which the force-displacement curves predicted by (3.5) best match the experimental data. Numerical results are given in Section 4.3. 


\subsection{Equations and Boundary Conditions}

We now formulate a boundary value problem corresponding to the capsule force-displacement experiments described in Section 2. The experimental situation has circular symmetry about the centre of the probe, and so we assume that the point $\boldsymbol{x}=$ $(R \cos \theta, R \sin \theta)$ in the $x_{3}=0$ plane is displaced to

$$
y=(r(R) \cos \theta, r(R) \sin \theta, z(R)) .
$$

The membrane equilibrium equations (3.4) thus reduce to a coupled system of nonlinear ordinary differential equations for the unknown functions $r$ and $z$. The boundary conditions (BCs) that we shall use are listed below.

\section{(i) At the probe:}

the capsule is assumed to stick to the probe, and so the BCs are taken to be

$$
\left.\begin{array}{l}
r(a)=a \\
z(a)=d
\end{array}\right\}
$$

(recall that as shown in Figure 2.1, the probe radius and displacement are $a$ and $d$ respectively).

(ii) At $R=A$ :

the capsule is clamped at $R=A$ and so the appropriate $\mathrm{BCs}$ are

$$
\left.\begin{array}{l}
r(A)=A \\
z(A)=0 .
\end{array}\right\}
$$

The BCs (i) are an approximation of the more physically realistic conditions that the tangential stress is zero and $z(R)=d$ for $r(R)<a$. We do not use these as boundary conditions because they convert the problem into a much more complicated inverse problem - these BCs depend on the value of the unknown solution $r(R)$.

A deformation of the type (4.1) with boundary conditions (4.2)-(4.3) has been considered by Roxburgh et al. (1995) for a Mooney-Rivlin membrane (see also Fulton and Simmonds (1986)). For this deformation the principal membrane stretches $\lambda_{\alpha}$ are

$$
\begin{aligned}
& \lambda_{1}(R)=\sqrt{\left(r^{\prime}\right)^{2}+\left(z^{\prime}\right)^{2}} \\
& \lambda_{2}(R)=r(R) / R
\end{aligned}
$$

where a prime ' denotes $d / d R$. It is also shown by Roxburgh et al. (1995) that the columns $\boldsymbol{T}^{\alpha}$ of the membrane stress $T$ are

$$
\begin{aligned}
& T^{1}=t_{1}\left\{\cos \eta\left(\begin{array}{c}
\cos \theta \\
\sin \theta \\
0
\end{array}\right)+\sin \eta\left(\begin{array}{l}
0 \\
0 \\
1
\end{array}\right)\right\} \text { and } \\
& T^{2}=t_{2} / R\left(\begin{array}{c}
-\sin \theta \\
\cos \theta \\
0
\end{array}\right),
\end{aligned}
$$

where

$$
\begin{aligned}
& r^{\prime}=\lambda_{1} \cos \eta, \\
& z^{\prime}=\lambda_{1} \sin \eta,
\end{aligned}
$$

and where $t_{\alpha}=\partial w / \partial \lambda_{\alpha}$ for $\alpha=1$, 2, i.e.

$$
\begin{aligned}
& t_{1}=\mu\left(\lambda_{1}-\lambda_{2}^{-2} \lambda_{1}^{-3}\right) e^{\gamma\left(I_{1}+1 / I_{2}-3\right)} \\
& t_{2}=\mu\left(\lambda_{2}-\lambda_{1}^{-2} \lambda_{2}^{-3}\right) e^{\gamma\left(I_{1}+1 / I_{2}-3\right)} .
\end{aligned}
$$

As shown by Roxburgh et al. (1995) the membrane equilibrium equations are thus

$$
\left(R t_{1} \cos \eta\right)^{\prime}=t_{2}
$$

and

$$
\left(R t_{1} \sin \eta\right)^{\prime}=0,
$$

and the second of these can be integrated to give

$$
R t_{1} \sin \eta=\Lambda
$$

where $\Lambda$ is a constant for all $R \in[a, A]$ (it depends only on the material parameters $\mu$ and $\gamma$ and on the probe displacement $d$ ).

The equations to be solved are then (4.5)-(4.10), and they can be written as the system

$$
\left.\begin{array}{l}
r^{\prime}=\lambda_{1} \cos \eta \\
z^{\prime}=\lambda_{1} \sin \eta \\
(\cot \eta)^{\prime}=t_{2} / \Lambda \\
t_{1}=\Lambda /(R \sin \eta)
\end{array}\right\}
$$

for $R \in(a, A)$, where $t_{1}$ and $t_{2}$ are the functions of $\lambda_{1}$ and $\lambda_{2}=r / R$ given in equations (4.7)-(4.8) above. 


\subsection{The Numerical Algorithm}

For given values of the parameters $\mu$ and $\gamma$ the system of equations (4.11) and boundary conditions (4.2)-(4.3) can be solved numerically by a shooting method using the algorithm described in (Roxburgh et al. 1995). The region $[a, A]$ is discretised into $N$ intervals with nodes $R_{j}=a+j h$ for $j=0: N$, where $h=(A-a) / N$, and we use the shorthand notation that $r_{j}=r\left(R_{j}\right), z_{j}=z\left(R_{j}\right)$ etc.. The solution strategy is outlined below.

\section{(1) Guess shooting parameter values.}

Guess values for $\eta\left(R_{0}\right)=\eta^{*}$ and $\lambda_{1}\left(R_{0}\right)=\lambda_{1}^{*}$.

(2) At $R=R_{0} \equiv a$.

The boundary conditions (4.2) give $z_{0}=d, r_{0}=a$ and hence $\lambda_{2}\left(R_{0}\right)=1$. Evaluate $\cos \eta_{0}, \sin \eta_{0}$ and $\cot \eta_{0}$ (using the fact that $\eta_{0}=\eta^{*}$ ). Substitute for $\lambda_{1} \equiv \lambda_{1}^{*}$ and $\lambda_{2}$ into (4.7)-(4.8) to calculate $t_{1}$ and $t_{2}$ at $R=R_{0}$, and finally compute $\Lambda=\left[R t_{1} \sin \eta\right]_{0}$. (3) Loop over $j=I: N$.

(i) Use Euler's method to numerically integrate the three ODEs $(4.11)_{1.2 .3}$ at $R_{j}$ to obtain

$$
\begin{aligned}
r_{j} & =r_{j-1}+h\left[\lambda_{1} \cos \eta\right]_{j-1} \\
z_{j} & =z_{j-1}+h\left[\lambda_{1} \sin \eta\right]_{j-1} \\
{[\cot \eta]_{j} } & =[\cot \eta]_{j-1}+h\left[t_{2}\right]_{j-1} / \Lambda
\end{aligned}
$$

(ii) Calculate $\cos \eta$ and $\sin \eta$ (from $\cot \eta$ ), $\lambda_{2}$ (from (4.4)) and $t_{1}$ (from (4.11)) $)_{4}$, all at $R=R_{j}$.

(iii) Use the computed value $t_{1}\left(R_{j}\right)$ to solve equation (4.7) numerically for $\lambda_{1}$ at $R_{j}$ (this is always possible because the material satisfies

$$
\begin{aligned}
\partial t_{1} / \partial \lambda_{1}= & \mu\left[1+3 \lambda_{1}^{-4} \lambda_{2}^{-2}+2 \gamma\left(\lambda_{1}-\lambda_{1}^{-3} \lambda_{2}^{-2}\right)^{2}\right] \\
& \times e^{\gamma\left(I_{1}+1 / I_{2}-3\right)}>0
\end{aligned}
$$

for all $\left.\left(\lambda_{1}, \lambda_{2}\right)\right)$. The solution can be computed using a nonlinear equation solving routine (for example the NAG routine C05AGF (Numerical Algorithms Group 1996)).

(iv) Use (4.8) to compute $t_{2}\left(R_{j}\right)$ from $\lambda_{1}$ and $\lambda_{2}$.

(4) At $\boldsymbol{R}=\boldsymbol{R}_{N} \equiv \boldsymbol{A}$.

Set $\boldsymbol{F}=\left(z_{N}, r_{N}-A\right)$.

(5) Check $F$.
If $\boldsymbol{F} \neq \boldsymbol{\theta}$ then adjust the shooting parameters $\eta^{*}$ and $\lambda_{1}^{*}$ and go back to (2). If $\boldsymbol{F}=\boldsymbol{0}$ (or more realistically, if $|\boldsymbol{F}|$ is less than a prescribed tolerance), then STOP.

If $\boldsymbol{F} \neq \boldsymbol{0}$ then the choice of shooting parameters $\eta^{*}$ and $\lambda_{1}^{*}$ does not solve the problem $(\boldsymbol{F}=\boldsymbol{0}$ corresponds to satisfying the boundary condition (4.3)), and so they have to be adjusted. If the above steps (2)-(4) are coded up into a subroutine that takes the vector $\left(\eta^{*}, \lambda_{1}^{*}\right)$ as input and computes and outputs the vector $\boldsymbol{F}$, then a nonlinear system solver (e.g. the NAG routine C05NBF (Numerical Algorithms Group 1996)) can be used to calculate values of $\left(\eta^{*}, \lambda_{1}^{*}\right)$ for which $|\boldsymbol{F}|$ is acceptably small. The solution $(r, z)$ for these shooting parameter values can then be computed.

In summary, the above steps can be used to calculate the deformation $(r, z)$ for any fixed value of the material parameters $\gamma$ and $\mu$. However, recall that what we are actually trying to do is to compute the values of these two parameters that best fit the experimental force-displacement data. We now describe how this may be achieved by incorporating steps (1)-(5) into a subroutine that is called by a nonlinear least squares routine (e.g. the NAG routine E04FDF (Numerical Algorithms Group 1996)). We begin by detailing the relationship between the deformation $(r, z)$ and the force acting on the probe.

Recall that the contact force per unit length across a material curve on the surface $S$ is

$$
\tau=T \nu,
$$

where $v$ is the outward unit normal to the curve lying in the tangent plane of $S$. This implies that the force exerted on the membrane by the probe at the point $(a \cos \theta, a \sin \theta, 0)$ is

$$
\tau=T\left(\begin{array}{c}
\cos \theta \\
\sin \theta \\
0
\end{array}\right) .
$$

As noted by Roxburgh et al. (1995) it follows that $\tau=T^{1}$, and hence (using (4.10)) that the normal component $\widehat{f}(\theta)$ of the force at the point is

$$
\widehat{f}(\theta)=t_{1} \sin \eta=\Lambda / a \text {. }
$$


The total normal force $f$ exerted by the probe on the material is $f=2 \pi a \widehat{f}(\theta)$, i.e.

$$
f=2 \pi \Lambda
$$

Suppose that we have the set of probe displacement and force measurements $\left\{\left(d_{k}, f_{k}\right)\right\}_{k=1}^{M}$. The predicted force at a given displacement $d$ is $f=$ $2 \pi \Lambda$, where $\Lambda$ depends (only) on $\gamma, \mu d$, and we also introduce an offset $\varepsilon$ to take account of any experimental errors due to the position at which the zero displacement is measured. The force predicted by the model can thus be written as

$$
f=2 \pi \Lambda(\gamma, \mu, d)+\varepsilon
$$

The least squares error between the model and the data is then

$$
\mathcal{E}(\gamma, \mu, \varepsilon)=\sum_{k=1}^{M}\left[2 \pi \Lambda\left(\gamma, \mu, d_{k}\right)+\varepsilon-f_{k}\right]^{2},
$$

and the best fit parameter values are those that minimise the error $\mathcal{E}$.

Using a least squares minimisation routine to compute the values of $\gamma, \mu$ and $\varepsilon$ that minimise $\mathcal{E}$ involves supplying a subroutine that takes the values of these parameters as input and outputs $\mathcal{E}$. This subroutine contains the steps (1)-(5) of the shooting method as an inner loop.

\subsection{Numerical Results}

Figure 2.3 shows that there is quite a wide variation in the mechanical properties of the spleen capsule samples tested. It therefore follows that there will also be a wide spread in the 'best fit' values of the parameters $\gamma$ and $\mu$ for all the samples. Consequently we shall show that (3.5) is a reasonable model for the initial loading of spleen capsule by just concentrating on a small number of data samples that represent the range of possible behaviour shown in Figure 2.3.

We shall use the three samples cap1, cap2 and cap3, obtained using a probe rate of $60 \mathrm{rpm}$ and shown in Figure 4.1.

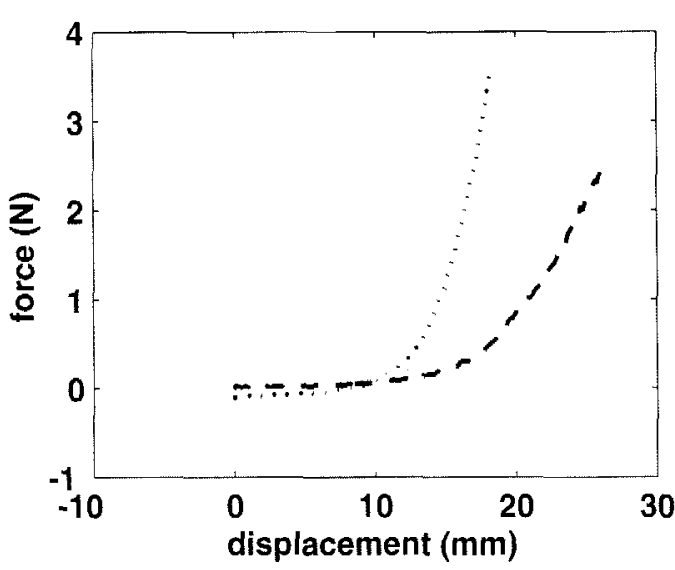

FIGURE 4.1 Force-displacement plots for the data in samples cap1 (dotted), cap2 (solid) and cap3 (dashed).

Each of these samples contains approximately 150 data points, and computing the best fit material parameters using all the points takes a considerable length of time (the computer time taken by the nonlinear least squares fitting program increases with the number of data points). We shall show that the computed values of $\gamma$ and $\mu$ are reasonably insensitive to the number of data points used, and that it is sufficient to use a small subset of the total data points.

Suppose that the experimental output for one sample is a collection of a large number of displacement and force values, with the maximum displacement being $d_{\text {max }}$. The errors inherent in the algorithm described in the previous subsection mean that there are reasonably large relative errors in the shooting method part of the calculation when the force is small (Euler's method is used because it is an explicit integrator: it is only first order accurate). We therefore ignore the first part of the data set and restrict our attention to points for which the displacement is at least $6 \mathrm{~mm}$. An $M$-point data subset $\left\{d_{k}, f_{k}\right\}_{k=1}^{M}$ is chosen by splitting the interval (6, $d_{\max }$ ) into $M-1$ (approximately equal) intervals by setting the $k$ th point $d_{k}$ to be the point closest to $6+(k-1)\left(d_{\max }-6\right) /(M-1)$ for $k=1: M$ (so $d_{1} \approx 6$ and $\left.d_{M}=d_{\text {max }}\right)$.

We have calculated the best fit values of $\mu$ and $\gamma$ and the offset $\varepsilon$ for each data sample when $M$ 
is 5, 10, 20 and 40, and the results are tabulated below. Note that the computed values of $\mu$ and $\gamma$ do not depend strongly on the number $M$ of data points used.

\begin{tabular}{rccc}
\hline \multicolumn{4}{c}{ cap1 } \\
\hline 5 & $\mu$ & $\gamma$ & $\varepsilon$ \\
\hline 5 & 0.168720 & 2.65365 & -0.127868 \\
20 & 0.171965 & 2.67081 & -0.135817 \\
40 & 0.174839 & 2.68764 & -0.137631 \\
& 0.178483 & 2.73955 & -0.137642 \\
\hline
\end{tabular}

\begin{tabular}{rccc}
\hline \multicolumn{4}{c}{ cap2 } \\
\hline 5 & $\mu$ & $\gamma$ & $\varepsilon$ \\
10 & 0.039103 & 0.88335 & -0.086700 \\
20 & 0.040929 & 0.87328 & -0.121056 \\
40 & 0.041384 & 0.91093 & -0.128896 \\
& 0.041375 & 0.97231 & -0.125062 \\
\hline
\end{tabular}
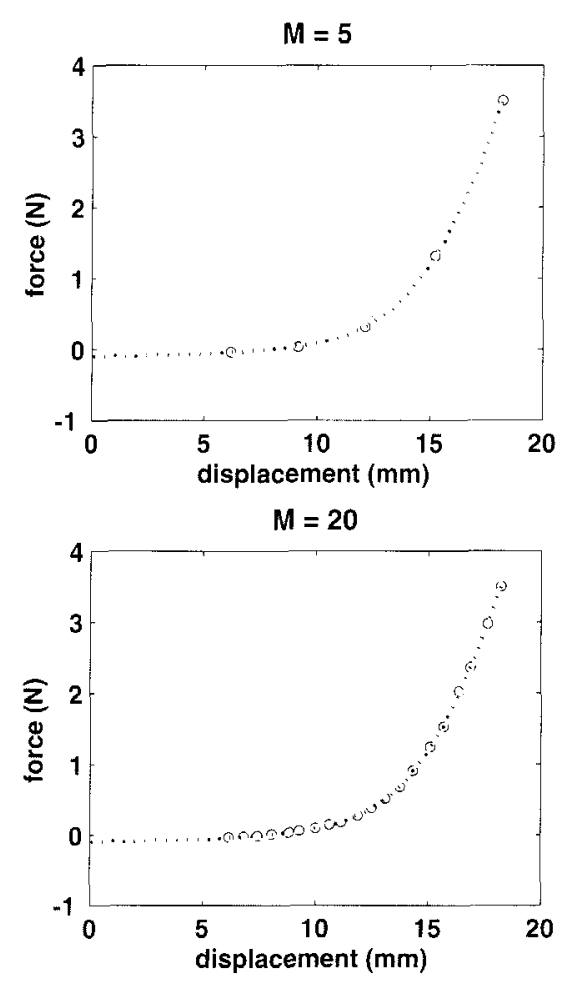

\begin{tabular}{rccc}
\hline \multicolumn{4}{c}{ cap3 } \\
\hline $\mathbf{5}$ & $\mu$ & $\gamma$ & $\varepsilon$ \\
10 & 0.018397 & 1.04770 & -0.023844 \\
20 & 0.019563 & 0.99298 & -0.055198 \\
40 & 0.019192 & 1.05071 & -0.051323 \\
& 0.019379 & 1.04237 & -0.054313 \\
\hline
\end{tabular}

Graphs of the full data set (dotted black), data points used (circles) and the force-displacement curves predicted by the model (3.5) with the best fit parameter values for each sample (solid grey) are shown in Figures 4.2-4.4.

The capsule deformation (i.e. the solution $(r, z)$ of the boundary value problem) is qualitatively the same for all the data samples. Figure 4.5 shows the computed capsule shape for cap2 at four values of the displacement $d_{k}$. This solution was calculated using the best fit parameters for the sample when $M=20$. Note the similarity to the deformations
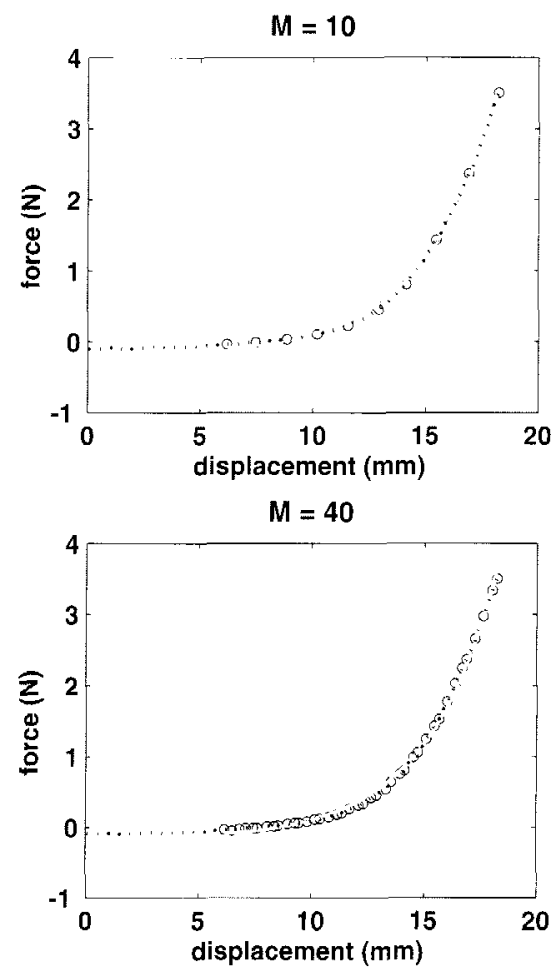

FIGURE 4.2 Force-displacement plots for various values of $M$ for sample cap1. The graphs show the full data set (black dotted), the $M$ data points ( $\left.d_{k}, f_{k}\right)_{k=1}^{M}$ used in the least squares routines (circles) and the prediction of the model (3.5) (solid grey). 

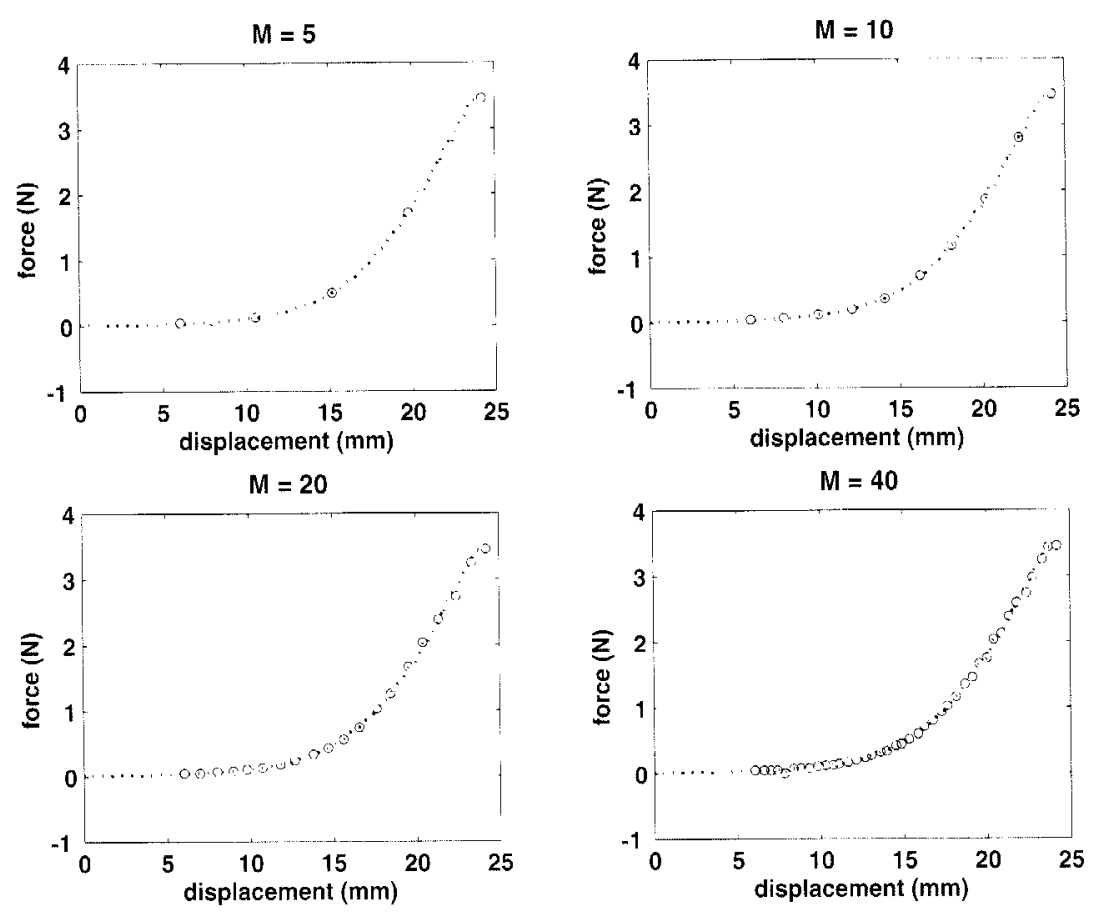

FIGURE 4.3 Force-displacement plots for various values of $M$ for sample cap2. The graphs show the full data set (black dotted). the $M$ data points $\left(d_{k}, f_{k}\right)_{k=1}^{M}$ used in the lcast squares routines (circles) and the prediction of the model (3.5) (solid grey).
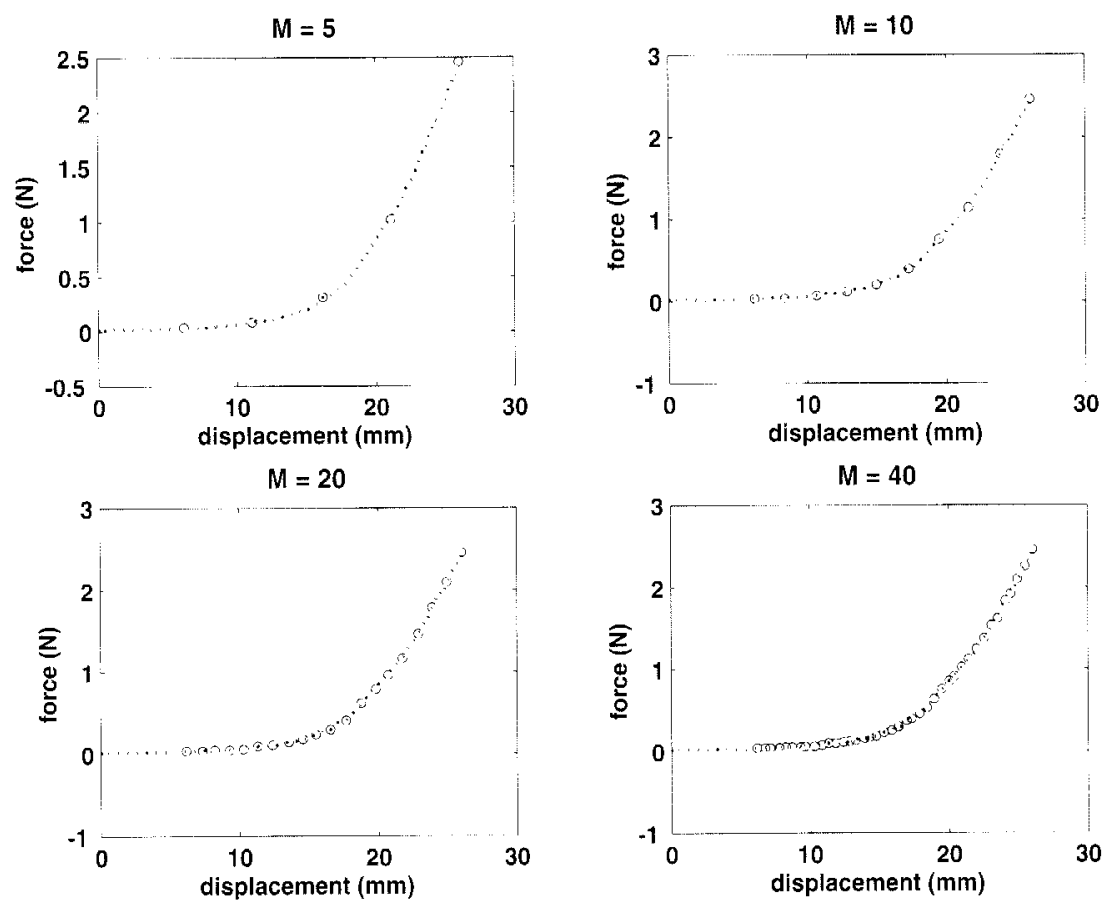

FIGURE 4.4 Force-displacement plots for various values of $M$ for sample cap3. The graphs show the full data set (black dotted), the $M$ data points $\left(d_{k}, f_{k}\right)_{k=1}^{M}$ used in the least squares routines (circles) and the prediction of the model (3.5) (solid grey). 

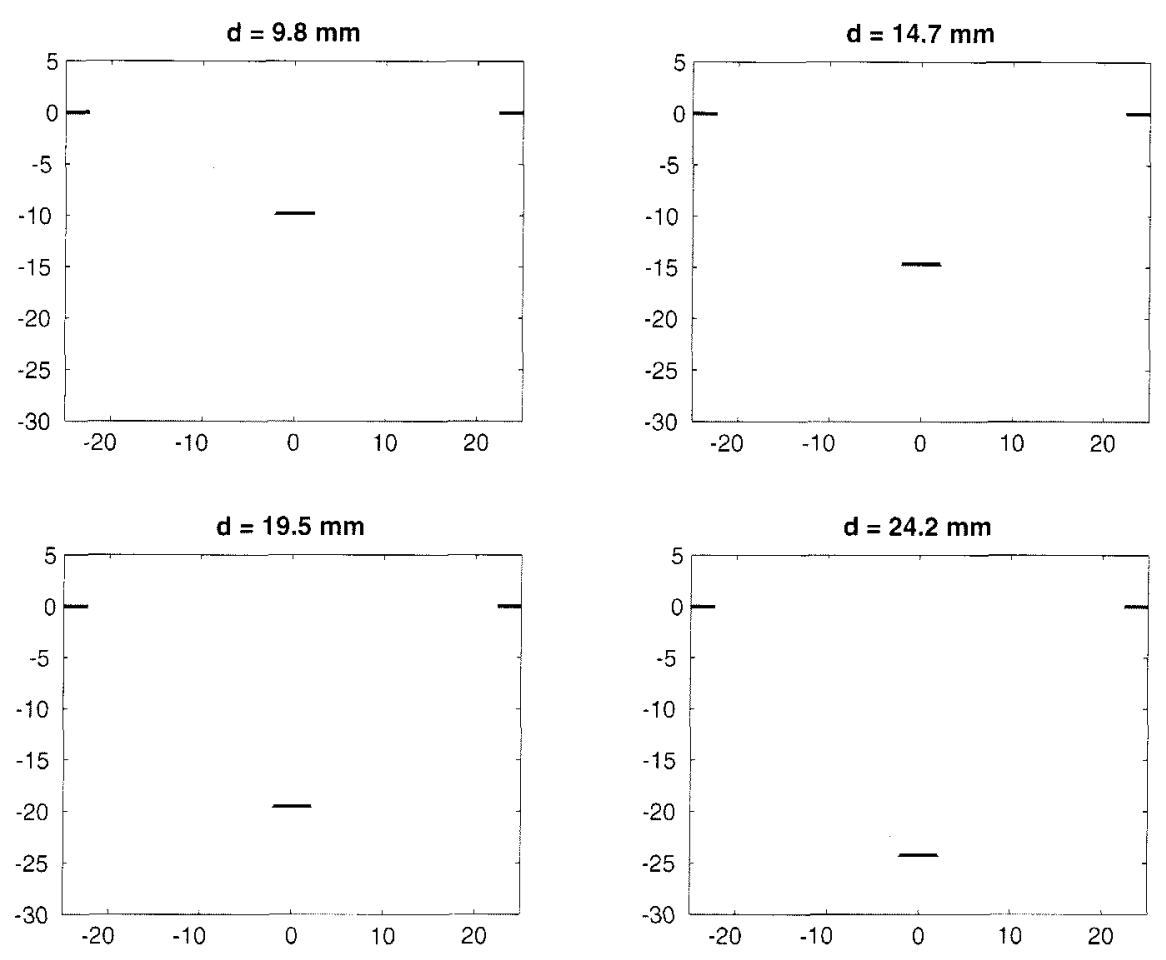

FIGURE 4.5 Computed capsule deformation for sample cap2 with $M=20$ at four values of the probe displacement $d$. All units used are $\mathrm{mm}$.

shown in (Fulton and Simmonds 1986, Roxburgh et al. 1995).

\section{DISCUSSION AND FURTHER WORK}

We have shown that the membrane model (3.5) gives a good representation of the mechanical behaviour of pig spleen capsule samples when they are first loaded. As illustrated by Figures 4.2-4.4 the computed best fit model matches the data very well (surprisingly well considering that the capsule is not really a membrane and that the boundary conditions (4.2) imposed at the probe are not totally realistic), and hence the model should be able to predict the response of capsule tissue over a wide range of deformations.

The long-term project objective is to develop sophisticated biomechanical models for the soft tissues and organs of the abdominal cavity which can be incorporated into software systems for electronic tissue simulations. Although vital in the development of such a model for the spleen, the work described here and in (Carter and Davies 1996) is just the start of the project. The next steps are to try to use the membrane model (3.5) to predict the amount of stress needed to puncture the capsule and to make comparisons with experimental data, and to consider a composite model consisting of the membrane (3.5) surrounding interior tissue of the type modelled by Carter and Davies (1996). We then aim to obtain suitable data from a small number of clinical measurements on living human spleen tissue (in patients undergoing splenectomy), and use it to adjust the pig spleen biomechanical models appropriately.

\section{Acknowledgements}

This work was supported by the Defense Advanced Research Projects Agency (contract no. MDA97295-1-0019). 


\section{References}

Atkin, R. J. and Fox, N. (1980) An Introduction to the Theory of Elasticity, Longman.

Beatty, M. F. (1987) Topics in finite elasticity: Hyperelasticity of rubber, elastomers, and biological tissues - with examples, Appl. Mech. Rev, , 40(12).

Brown, W., Satava, R. and Rosen, J. (1994) Virtual reality and surgical training: simulating the future, Minimally Invasive Therapy, 3, 81-86.

Carter, F., Russell, E., Dunkley, P. and Cuschieri, A. (1994) Restructured animal tissue model for training in laparoscopic anti-reflux surgery, Minimally Invasive Therapy, 3, 87-94.

Carter, F. J. and Davies, P. J. (1996) Biomechanical and physical modelling of the handling characteristics of tissues and organs during surgical interventions. (ARPA progress reports), University of Dundee.

Demiray, H. (1972) A note on the elasticity of soft biological tissues, J. Biomechanics, 5, 309-311.

Demiray, H. (1981) Large deformation analysis of some soft biological tissue, Journal of Biomechanical Engineering, 103, 73-78.

Demiray, H. (1983) Incremental elastic modulus for isotropic elastic bodies with application to arteries, Journal of Biomechanical Engineering, 105, 308-309.

Fulton, J. P. and Simmonds, J. G. (1986) Large deformations under vertical edge loads of annular membranes with various strain energy densities, Int. J. Non-Linear Mechanics, 21, 257-267.

Fung, Y. C. B. (1967) Elasticity of soft tissues in simple elongation, American Journal of Physiology, 213, 1532-1544.

Fung, Y. C. (1993) Biomechanics: mechanical properties of living tissues, second edn, Springer-Verlag, New York.

Green, A. E., Naghdi, P. M. and Wainwright, W. L. (1965) A general theory of a Cosserat surface, Arch. Rat. Mech. Analysis, 20, 287-308.

Gurtin, M. E. (1983) Topics in Finite Elasticity, SIAM.
Humphrey, J. D. and Yin, F. C. P. (1989) Biomechanical experiments on excised myocardium: theoretical considerations, $J$. Biomechanics, 22, 377-383.

Naghdi, P. M. (1972) Theory of shells and plates, in S Flügge (ed.), Handbuch der Physik vol. Vla/2, Springer-Verlag, Berlin, pp. 425-640.

Numerical Algorithms Group (1996) Fortran Library (Mark 17) (NAG Ltd., Oxford, UK, OX2 8DR).

Ogden, R. W. (1984) Non-linear Elastic Deformations, Wiley, New York.

Rivlin, R. S. and Saunders, D. W. (1951) Large clastic deformations of isotropic materials vii. experiments on the deformation of rubber, Phil. Trans. A, 243, 251-288.

Roxburgh, D. G., Steigmann, D. J. and Tait, R. J. (1995) Azimuthal shearing and transverse deflection of an annular elastic membrane, International Journal of Engineering Science, 33, $27-43$.

Satava, R. M. (1993) Virtual reality surgical simulator, Surgical Endoscopy, 7, 203-205.

Trowbridge, E. A., Black, M. M. and Daniel, C. L. (1985) The mechanical response of glutaraldehyde-fixed bovine pericardium, Journal of Materials Science, 20, 114-140.

Trowbridge, E. A. and Crofts, C. E. (1987) The extension rate independence of the hysteresis in glutaraldehyde-fixed bovine pericardium, Biomaterials, 8, 201-206.

Wang, B. C., Wang, G. R., Yan, D. H. and Liu, Y. P. (1992) An experimental study on biomechanical propertics of hepatic tissue using a new measuring method, Bio-Medical Materials and Engineering, 2, 133-138.

Zioupos, P., Barbenel, J. C., Fisher, J. and Wheatley, D. J. (1993) Changes in the mechanical properties of bioprosthetic valve leaflets made of bovine pericardium, as a result of longterm mechanical conditioning in vitro and implantation in vivo, Journal of Materials Science: Materials in Medicine, $\mathbf{4}$ 531-537.

Zioupos, P. and Barbenel, J. C. (1994) Mechanics of native bovine pericardium II, Biomaterials, 15, 374-382. 


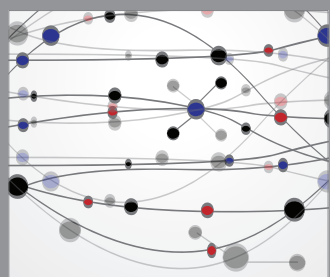

The Scientific World Journal
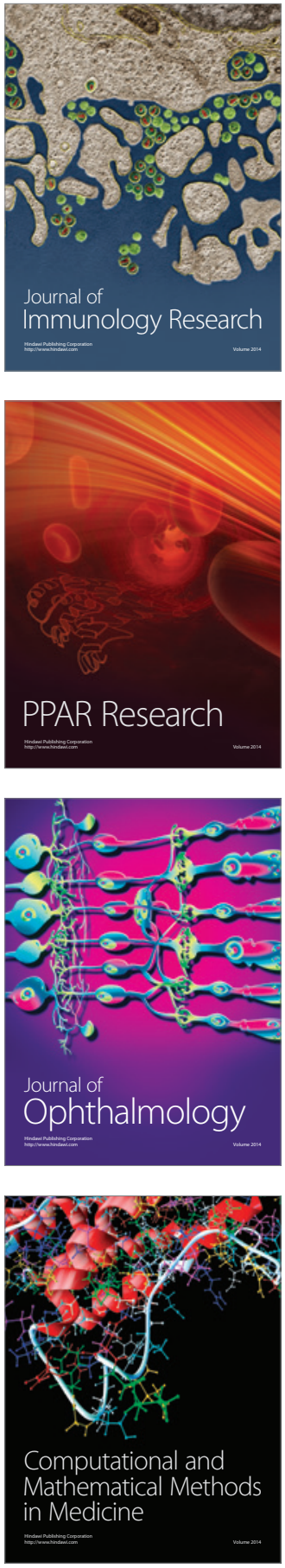

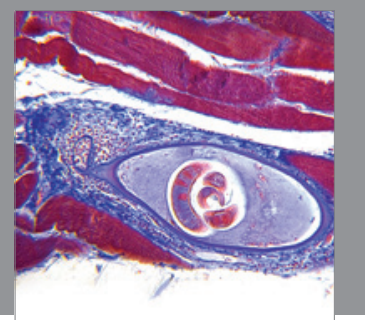

Gastroenterology

Research and Practice
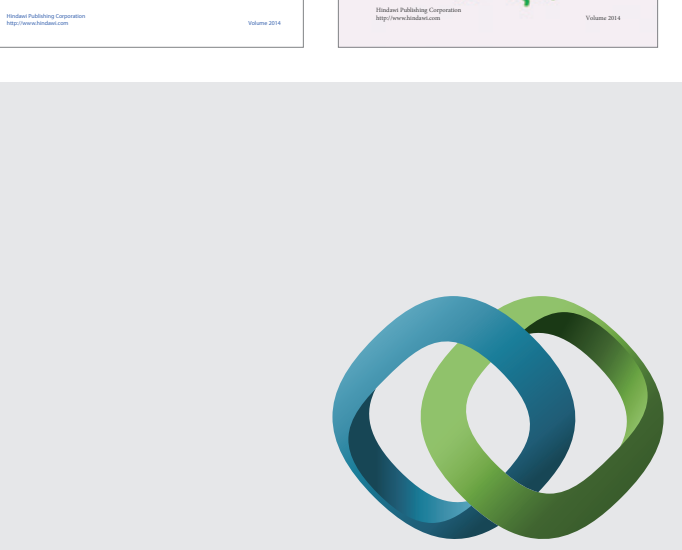

\section{Hindawi}

Submit your manuscripts at

http://www.hindawi.com
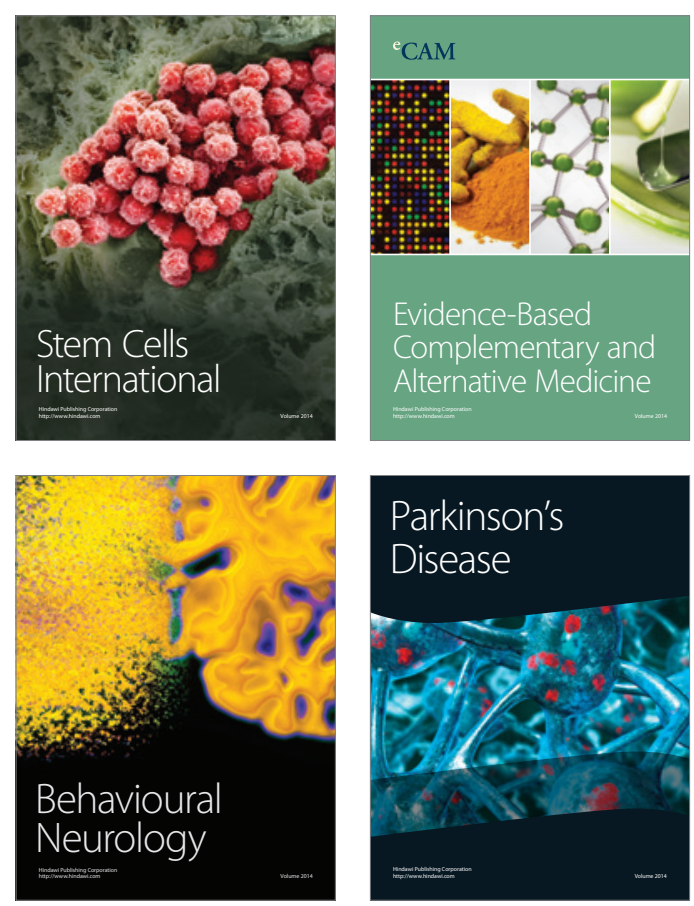

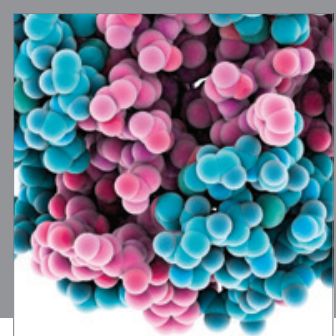

Journal of
Diabetes Research

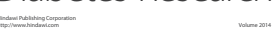

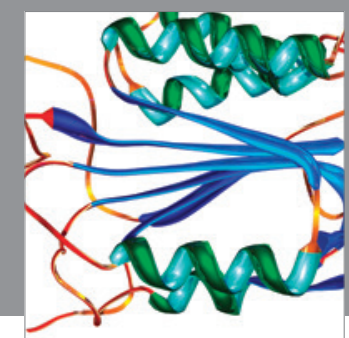

Disease Markers
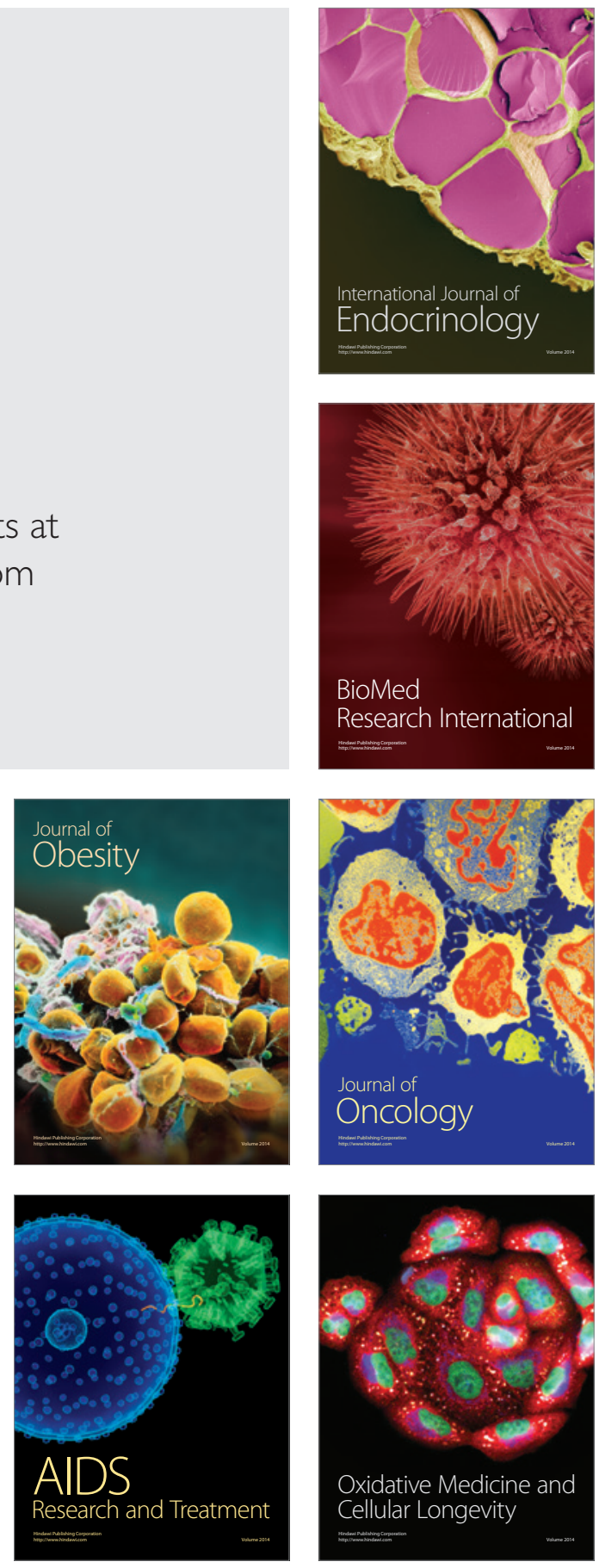